\title{
Les lémuriens du site Ramsar de Torotorofotsy
}

\author{
Gilbert Rakotondratsimba', Rosette Ralisoamalala' et \\ Jonah Henri Ratsimbazafy'
}

\section{RÉSUMÉ}

Les différentes ressources naturelles et potentialités économiques du marais de Torotorofotsy et de ses bassins versants justifient l'appellation de "Ramsar » du site. Ce dernier abrite plusieurs espèces menacées de disparition: des amphibiens (Mantella aurantiaca et $M$. crocea), des oiseaux (Anas melleri, Sarothrura watersi et Tyto soumagnei), des mammifères carnivores (Cryptoprocta ferox) ainsi que des lémuriens. La présente étude stipule qu'il y existe treize espèces de lémuriens ; parmi ces espèces, six sont classées «En danger » (Prolemur simus, Indri indri, Propithecus diadema, Hapalemur griseus, Eulemur rubriventer, Daubentonia madagascariensis), une "Quasi menacée » (Eulemur fulvus), une "Non menacée » (Avahi laniger) et cinq "Non évaluées" (Allocebus trichotis, Cheirogaleus major, C. crossleyi, Microcebus lehilahytsara et Lepilemur mustelinus). Les potentialités économiques de cette localité (ressources forestières, terrains agricoles et ressources minières) incitent à l'immigration dont on peut déjà voir des conséquences sur une partie de Torotorofotsy et de ses bassins versants : exploitation forestière, transformation de la forêt en tavy et altération des marais en zones agricoles. L'implantation du projet d'extraction de nickel-cobalt d'Ambatovy à l'ouest du site a mené à la construction d'un pipeline traversant le marais, ce qui facilite l'accès au site mais amplifie la pression anthropique sur l'environnement et entraine la dégradation de cette biodiversité particulière à la région. Des études environnementales et socio-économiques ont été effectuées pour réajuster le plan de gestion et de conservation du site. Pour ce qui est des lémuriens, les études de pression et de menaces basées sur la méthodologie du 'The Nature Conservancy' et celle de 'la Conservation du site Ramsar', focalise les efforts de conservation, pour ce site, sur six espèces à haut risque de disparition : Prolemur simus, Propithecus diadema, Indri indri, Eulemur rubriventer, Daubentonia madagascariensis et Allocebus trichotis.

\section{ABSTRACT}

The Torotorofotsy wetlands Ramsar site is rich in natural resources and has great economic potential. Several threatened species, including amphibians (Mantella aurantiaca and

\author{
Correspondence: \\ Jonah Henri Ratsimbazafy \\ Groupe d'Étude et de Recherche sur les Primates de Madagascar \\ Madagscar \\ E-mail: jonah.ratsimbazafy@durrell.org
}

M. crocea), birds (Anas melleri, Sarothrura watersi and Tyto soumagnei), carnivores (Cryptoprocta ferox) and lemurs distinguish the site. This study documents the presence of thirteen species of lemur, of which six are considered threatened (Prolemur simus, Hapalemur griseus, Eulemur rubriventer, Propithecus diadema, Indri indri and Daubentonia madagascariensis), one is classed Near Threatened (Eulemur fulvus), one Least Concern (Avahi laniger) and five Data Deficient (Allocebus trichotis, Cheirogaleus major, C. crossleyi, Microcebus lehilahytsara and Lepilemur mustelinus). The economic potential (forest resources, agricultural land and mineral resources) of the site has stimulated immigration to the area. As a result one part of Torotorofotsy has been subjected to forest exploitation, some areas of forest have been transformed by tavy and a large part of the wetlands has come under cultivation. The nickel and cobalt exploitation by the Ambatovy Project is located to the west of Torotorofotsy, thus one part of the pipeline runs through the marshland facilitating access to the site. The attraction and migration of human populations to the site generates pressures on the environment and subsequently accelerates the degradation of its biodiversity. Several studies based on the methodology of the 'The Nature Conservancy' and 'the Conservation of Ramsar site' have been conducted in order to implement and revise the management plan of this site. At Torotorofotsy, lemur conservation efforts should be focused on the six species at higher risk: Prolemur simus, Propithecus diadema, Indri indri, Eulemur rubriventer, Daubentonia madagascariensis and Allocebus trichotis.

\section{INTRODUCTION}

En 2008, le Projet minier d'Ambatovy a apporté son appui à la mise à jour du plan d'aménagement du marais de Torotorofotsy et de ses bassins versants, dans le cadre de sa politique de gestion durable de ce site Ramsar. Cette initiative de conservation à été mise en place dans le cadre de l'engagement au "Projet Ambatovy sur I'environnement à Madagascar » et selon les exigences du texte sur la mise en compatibilité des investissements sur l'environnement (MECIE : décret n²004-167 du 03 février 2004). Ce projet est engagé depuis 2006, depuis la signature de l'accord tripartite entre l'État Malgache, représenté 
par le Ministère de l'Environnement des Eaux et Forêts, I'Association Mitsinjo et la Société Ambatovy pour le pilotage du site. Il semble important de rappeler que le marais de Torotorofotsy et ses bassins versants ont été déclarés "site Ramsar ", le 2 février 2005 et que l'institution non-gouvernementale "Association Mitsinjo » est, depuis le 22 juillet 2005, I'entité responsable de la mise en œuvre du plan de gestion du site (Attestation n487-05/MINENVEF/SG/DGEF/DPB/SCBLF du 22.07.2005). Ainsi, la mise en place de la présente étude $s^{\prime}$ inscrit à la fois dans I'amélioration de ce plan de conservation auquel nous avons ici appliqué le concept du 'The Nature Conservancy' (TNC 2003) et dans les modèles types de conservation de site Ramsar (Ramsar 1999).

À Madagascar, les lémuriens sont parmi les animaux clés du système de conservation de la biodiversité (Ganzhorn et al. 1997). Dans le souci de la conservation de ces animaux, dans le marais de Torotorofotsy et de ses bassins versants, nous avons comme objectifs, dans cette étude, d'identifier les lémuriens existant dans le site, d'analyser les menaces anthropiques qui peuvent influencer le dynamisme de leur population et de formuler des suggestions sur le recadrage de leur conservation en sauvegardant leurs habitats naturels, dans le concept de I'utilisation rationnelle des ressources naturelles actuelles par les populations locales.

TOROTOROFOTSY ET SES BASSINS VERSANTS.

Administrativement, Torotorofotsy est localisé dans la Région d'Alaotra-Mangoro, à une vingtaine de kilomètres au nord-est de Moramanga (Figure 1), à cheval sur trois communes rurales avec Andasibe à l'est, Ambohibary à l'ouest et Ampasipotsy au sud. Torotorofotsy et ses bassins versants ont une superficie de 9776 ha, dont 1100 ha sont formés par des marais et le reste est constitué par des collines recouvertes de forêt intacte humide sempervirente (Ministère de l'environnement et des forêts 2009). Les altitudes de ces reliefs varient entre 900 et $1200 \mathrm{~m}$. La qualité et la structure forestière diffèrent en fonction des facteurs édaphiques et de leurs dégradations; elles sont parfois fragmentées par des formations secondaires appelées savoka et des zones herbeuses arborées (Green et Sussman 1990, Rasoavarimanana 1997). Les études fauniques antérieures ont identifié 334 espèces de vertébrés, présentant un taux d'endémisme de plus de $90 \%$ Les poissons avec les Rheocles sp. et Ratsirakia sp., les amphibiens avec les Mantella aurantiaca et M. crocea, les oiseaux avec Anas melleri, Sarothrura watersi et Tyto soumagnei, les mammifères comme Cryptoprocta ferox et les lémuriens avec Daubentonia madagascariensis, Eulemur rubriventer, Indri indri, et Propithecus diadema étaient considérés comme les animaux inventoriés les plus rares (Zimmermann et Andrianarivo 2000).

\section{MÉTHODOLOGIE}

Étant donnée l'étendue de l'aire de Torotorofotsy et de ses bassins versants (9 776 ha), trois sites ont été retenus pour nos études, en considérant la densité de la couverture forestière. Le premier site d'étude $\mathrm{SE}_{1}$ est localisé dans la partie nord et nord-ouest ; le second site $\mathrm{SE}_{2}$ se trouve dans la zone nord-est et le troisième site $\mathrm{SE}_{3}$ dans la zone sud-ouest (Figure 1).

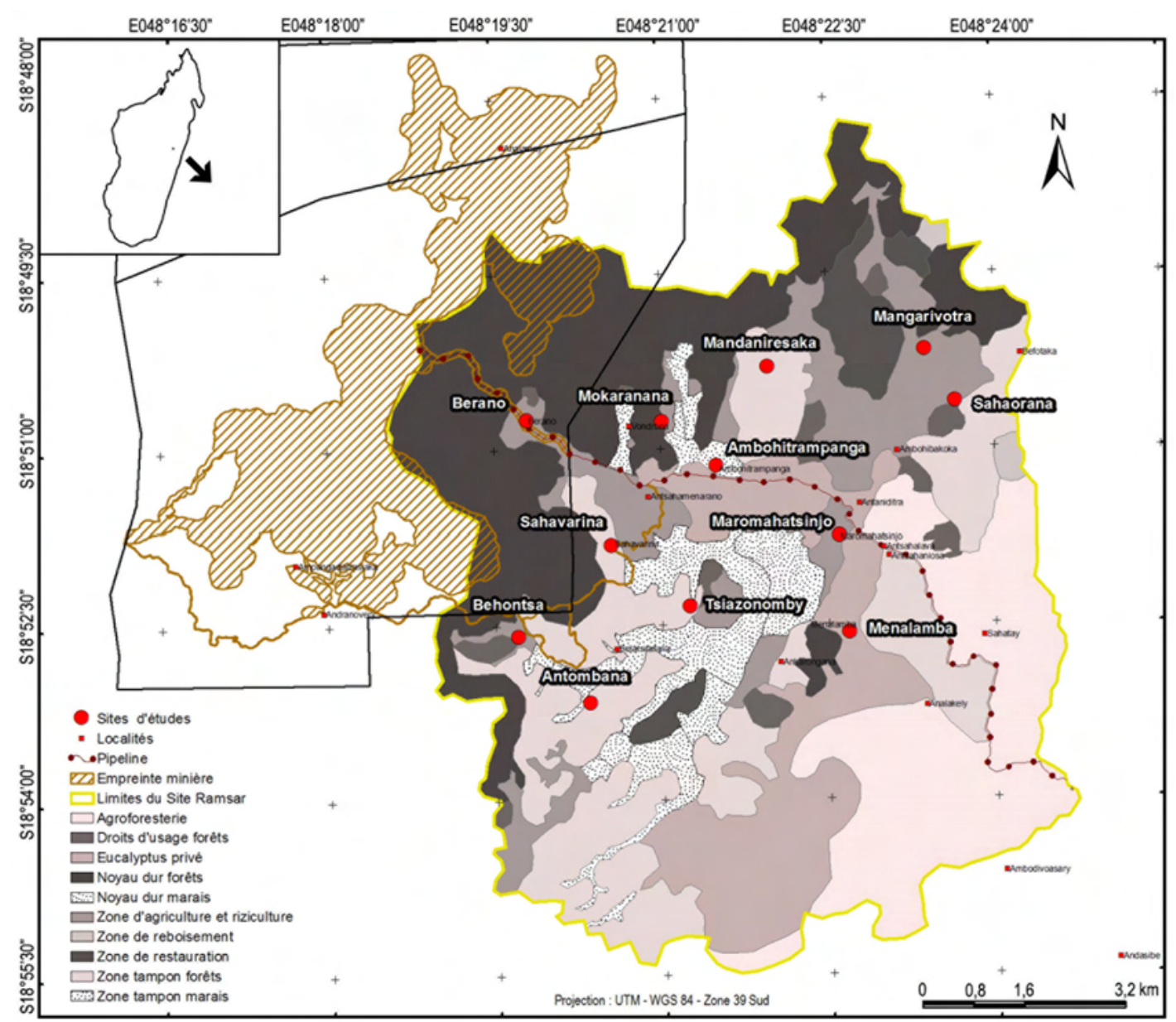

FIGURE 1. Localisation de Torotorofotsy et des sites d'études (Source : Département Environnement Ambatovy ; Site SE : Mandaniresaka, Mokaranana ; site $\mathrm{SE}_{2}$ : Mangarivotra, Sahaorana; site SEз : Antombana, Behontsa, Sahavarina) 
INVENTAIRE DES LÉMURIENS. La méthode de comptage utilisée se base sur I'utilisation d'une ligne " transect » le long de laquelle sont inventoriés les lémuriens. La longueur de chaque transect est d'environ $2000 \mathrm{~m}$. Les observations sont effectuées entre 0700-1000h, 1430-1730h et 1830-2130h. Par soucis d'exactitude, un minimum de trois transects a été installé sur chaque site afin de prendre en compte la variation des observations de l'échantillon. Au total, dix transects ont été placés : trois dans le site $\mathrm{SE}_{1}$, quatre dans le site $\mathrm{SE}_{2}$ et trois dans le site $\mathrm{SE}_{3}$ (Figure 1). Le même protocole d'observation a été adopté pour chaque transect : pendant sept jours et sept nuits d'inventaire, chaque observateur se déplace à une vitesse comprise entre 0,8 et $1 \mathrm{~km}$ par heure et à chaque fois qu'il rencontre un groupe d'animaux, il s'arrête et note l'heure de rencontre, la position géographique, le nombre d'individus rencontrés, ainsi que leurs âges relatifs et sexes et la distance perpendiculaire entre le premier animal observé et le transect. Les arrêts ne doivent pas dépasser cinq minutes. Les observateurs utilisent, pendant la nuit, des lumières artificielles atténuées (lampes frontales), qui facilitent le repérage des lémuriens; une fois un individu repéré, l'utilisation de lumières plus puissantes pendant quelques minutes est indispensable pour identifier l'espèce. Enfin, le sens dans lequel est parcouru chaque transect est inversé tous les jours afin de favoriser le hasard de l'échantillonnage. Les résultats de ces observations permettent d'estimer la densité des lémuriens dans chaque site, à partir du calcul adopté et employé par Ganzhorn (1994) et Schmid (2000), suivant l'équation : $D=N /(2 \times W \times L)$ avec $D$ : densité estimée de la population (individus $/ \mathrm{km}^{2}$ ou i $/ \mathrm{km}^{2}$ ) ; N : nombre total de lémuriens rencontrés pendant les observations (individus) ; W : distance perpendiculaire des animaux observés par rapport au transect en $\mathrm{km} ; \mathrm{L}$ : Iongueur totale du transect en $\mathrm{km}$. Avec cette méthode de calcul, nous avons exclu les animaux qui se trouvaient à une distance de plus de 15 mètres, afin d'optimiser l'identification des espèces et de limiter les erreurs. Le but de ces inventaires est de constater la présence ou l'absence des espèces de lémuriens et d'apprécier la densité relative des espèces existantes, afin de déterminer l'abondance des espèces cibles à conserver et d'évaluer, par la suite, leur viabilité à partir des études des facteurs écologiques. Des observations éventuelles en dehors de ces transects ont été effectuées pour évaluer la présence ou l'absence des espèces de lémuriens sur la totalité du site de Torotorofotsy.

MÉTHOdE D'ENQUÊTE SOCIO-ÉCONOMIQUE EN

RELATION AVEC L'ENVIRONNEMENT. Dans I'optique d'analyser la relation entre les pressions anthropiques et la conservation des lémuriens, nous avons adopté la « Méthode Accélérée de Recherche Participative de type exploratoire » ou "MARP exploratoire » (Gueye et Schoonmaker Freudenberger 1991). Cette méthode permet d'évaluer l'importance dans l'étude socio-écologique et sociobiologique de la zone de Torotorofotsy. Huit villages représentatifs de la zone ont été choisis : Ambohitrampanga, Mokaranana et Berano dans le site $\mathrm{SE}_{1}$, Menalamba, Mangarivotra et Maromatsinjo dans le site $\mathrm{SE}_{2}$ puis Behontsa et Sahavarina dans le site $\mathrm{SE}_{3}$. Les thèmes ont été orientés et structurés autour des activités paysannes pour comprendre la signification de la gestion des ressources naturelles pour ces populations. De ce fait, les questionnaires étaient cadrés sur les activités quotidiennes des ménages, sur I'utilisation des ressources forestières (exploitation du bois, des produits végétaux, collecte de miel, chasse et consommation d'animaux sauvages), sur le système d'accaparement des terrains pour l'agriculture (défrichement, champs de culture, utilisation du feu, installation de campements temporaires appelé lasy ou de campements permanent appelés potro), sur les exploitations minières dans la forêt ainsi que sur les autres utilisations de cette dernière (pacages des bétails, cimetière).

ÉVALUATION DE LA PRESSION ANTHROPIQUE. NOUS avons appliqué une approche participative par groupe focal et des interviews semi-structurés dans le cadre de la méthode MARP (Gueye et Schoonmaker Freudenberger 1991), pour évaluer l'ampleur de l'impact des activités quotidiennes des populations sur les ressources naturelles exploitées ; les résultats issus de cette méthode permettent de compléter les informations scientifiques exploitées en vue de la conservation des espèces de lémuriens menacés dans le site et de "valoriser " les pressions anthropiques existantes. Dans notre cas, nous avons estimé la relation entre la densité des lémuriens dans le bloc forestier de Torotorofotsy et les pressions anthropiques qui peuvent affecter ces abondances. C'est à partir de ces densités que l'on déduit l'indice de stabilité de chaque espèce de lémurien en fonction de la moyenne des rangs de ces densités relatives. La relation suivante permet d'effectuer le calcul de cette moyenne $(\mathrm{M}): \mathrm{M} \cdot=\sum_{\mathrm{i}=1}^{\mathrm{n}} \mathrm{x}_{\mathrm{i}}+\sum_{\mathrm{i}=1}^{\mathrm{n}} \mathrm{y}_{\mathrm{i}} / \sum_{\mathrm{i}=1}^{\mathrm{n}} \mathrm{x}_{\mathrm{i}}+\mathrm{y}_{\mathrm{i}}$

et les valeurs calculées correspondent à l'indice de stabilité ; où $x_{i}$ correspond aux différentes espèces étudiées dans le site et yi correspond aux différentes localités d'études (Martin and Bateson 1993). Dans cette étude, I'indice est dit « plus stable», si la valeur de $M \in[12 ; 10]$; I'indice est dit « stable», si la valeur de $M \in[9,99 ; 8]$; cet indice est dit «peu stable» si la valeur de $M \in[7,99 ; 3]$ et l'indice est dit «moins stable » si la valeur de $M \in[2,99 ; 0]$.

STRATÉGIE ET PRIORISATION DES ACTIONS DE

CONSERVATION. Les critères d'identification des zones prioritaires de conservation à Torotorofotsy et ses bassins versants sont établis à partir de la présence de pression et du rang de stabilité de l'espèce; c'est à dire en fonction de I'aire de distribution, de l'abondance relative des espèces, de la qualité de I'habitat et du niveau de menace qui pèse sur les lémuriens (TNC 2003). En effet, les sites prioritaires en matière de conservation des lémuriens à Torotorofotsy sont ceux qui présentent un taux élevé d'espèces menacées.

\section{RÉSULTATS}

LES LÉMURIENS DANS LA ZONE DE TOROTOROFOTSY ET SES BASSINS VERSANTS. Les résultats des inventaires dans les trois sites démontrent que la zone de Torotorofotsy et de ses bassins versants abrite 13 espèces de lémuriens parmi lesquelles six sont menacées, comme Prolemur simus, classé "En danger critique d'extinction» (CR) ; Indri indri, Propithecus diadema et Daubentonia madagascariensis catégorisées "En danger» (EN) (Mittermeier et al. 2010); Hapalemur griseus et Eulemur rubriventer classés «Vulnérable " (VU); une espèce a le statut de "Quasi-menacée » (NT), c'est le cas d'Eulemur fulvus; une espèce est classée "Préoccupation mineure » (LC), c'est le cas d'Avahi laniger. Enfin Allocebus trichotis, Cheirogaleus major, C. crossleyi, Microcebus lehilahytsara et Lepilemur mustelinus n'ont pas réellement de statut de conservation et désignées par «Données insuffisantes » (DD) (Tableau 1). 


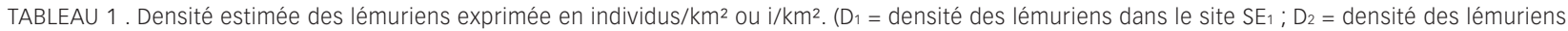
dans le site $\mathrm{SE}_{2} ; \mathrm{D}_{3}=$ densité des lémuriens dans le site $\mathrm{SE}_{3} ; \mathrm{NC}=$ non calculé - statut de conservation des espèces de lémuriens suivant UICN (2009) : CR = En danger critique d'extinction ; EN = En danger ; VU = Vulnérable ; NT = Quasi menacé $; \mathrm{LC}=$ Préoccupation mineure ; $\mathrm{DD}=\mathrm{Données}$ insuffisantes; $\mathrm{NE}=$ Non évalué).

\begin{tabular}{|c|c|c|c|c|c|c|c|c|c|}
\hline Famille & $\begin{array}{l}\text { Noms } \\
\text { scientifiques }\end{array}$ & $\mathrm{D} 1\left(\mathrm{i} / \mathrm{km}^{2}\right)$ & D2 (i/km²) & D3 $\left(\mathrm{i} / \mathrm{km}^{2}\right)$ & $\begin{array}{l}\text { Statut } \\
\text { UICN } \\
2010\end{array}$ & $\begin{array}{c}\text { Densité } \\
\left(\mathrm{i} / \mathrm{km}^{2}\right) \\
\text { dans les } \\
\text { autres aires } \\
\text { protegées }\end{array}$ & Localité & $\begin{array}{l}\text { Domaine } \\
\text { territorial } \\
\text { (ha) }\end{array}$ & Référence \\
\hline Cheirogaleidae & $\begin{array}{l}\text { Microcebus } \\
\text { lehilahytsara }\end{array}$ & $4,52 \pm 1,75$ & $2,31 \pm 1,64$ & $2,53 \pm 2,12$ & $\mathrm{DD}$ & & & & \\
\hline Cheirogaleidae & $\begin{array}{l}\text { Cheirogaleus } \\
\text { major }\end{array}$ & $1,64 \pm 0,9$ & 0 & 0 & $\mathrm{DD}$ & $(75-100)$ & Andasibe & $2-4$ & Pollock 1979 \\
\hline Cheirogaleidae & $\begin{array}{l}\text { Cheirogaleus } \\
\text { crossleyi }\end{array}$ & 0 & $0,46 \pm 0,5$ & $0,44 \pm 0,6$ & DD & & & & \\
\hline Cheirogaleidae & $\begin{array}{l}\text { Allocebus } \\
\text { trichotis }\end{array}$ & $0,41 \pm 0,5$ & 0 & 0 & DD & $(7-15)$ & Makira & & $\begin{array}{l}\text { Rasolofoson et al. } \\
2007 b\end{array}$ \\
\hline Lepilemuridae & $\begin{array}{l}\text { Lepilemur } \\
\text { mustelinus }\end{array}$ & $1,23 \pm 0,84$ & $0,92 \pm 0,9$ & $0,14 \pm 0,75$ & $\mathrm{DD}$ & & & $1-5$ & $\begin{array}{l}\text { Ratsirarson et al. } \\
1988\end{array}$ \\
\hline Lemuridae & $\begin{array}{l}\text { Eulemur } \\
\text { fulvus }\end{array}$ & $32,05 \pm 10,45$ & $13,83 \pm 8,35$ & $5,22 \pm 5,76$ & NT & $(40-60)$ & Andasibe & $7-20$ & Pereira et al. 1990 \\
\hline Lemuridae & $\begin{array}{l}\text { Eulemur } \\
\text { rubriventer }\end{array}$ & 0 & $1,84 \pm 0,9$ & 0 & VU & $(20-30)$ & Ranomafana & $10-20$ & Irwin et al. 2005 \\
\hline Lemuridae & $\begin{array}{l}\text { Prolemur } \\
\text { simus }\end{array}$ & NC & NC & 0 & $\mathrm{CR}$ & & & & \\
\hline Lemuridae & $\begin{array}{l}\text { Hapalemur } \\
\text { griseus }\end{array}$ & $6,99 \pm 5,44$ & $15,22 \pm 6,35$ & $1,64 \pm 1,25$ & VU & $(47-62)$ & Andasibe & 6-15 & Pollock 1979 \\
\hline Indriidae & Avahi laniger & $5,75 \pm 2,75$ & $13,37 \pm 2,55$ & $8,5 \pm 4,45$ & $\mathrm{LC}$ & $(72-100)$ & Andasibe & $2-4$ & Ganzhorn 1988 \\
\hline Indriidae & $\begin{array}{l}\text { Propithecus } \\
\text { diadema }\end{array}$ & 0 & $2,31 \pm 1,25$ & $0,44 \pm 0,75$ & EN & $(9-17)$ & Mantadia & $20-35$ & Schmid 2000 \\
\hline Indriidae & Indri indri & $4,11 \pm 2,63$ & $1,38 \pm 0,84$ & $0,9 \pm 0,9$ & EN & $(2-15)$ & Andasibe & $7-10$ & $\begin{array}{l}\text { Pollock 1979, } \\
\text { Powzyk et al. } 2003\end{array}$ \\
\hline \multirow[t]{2}{*}{ Daubentoniidae } & $\begin{array}{l}\text { Daubentonia } \\
\text { madagas- } \\
\text { cariensis }\end{array}$ & $0,82 \pm 0,77$ & NC & NC & EN & $(0-3)$ & Makira & $30-200$ & $\begin{array}{l}\text { Sterling 2003, } \\
\text { Rasolofoson et al. } \\
2007 b\end{array}$ \\
\hline & $\begin{array}{l}\text { Total } \\
\text { (espèces } \\
\text { existantes) }\end{array}$ & 10 & 11 & 9 & & & & & \\
\hline
\end{tabular}

À noter que pendant nos observations en dehors des transects, nous avons recensé un groupe de six individus de Prolemur simus près du village de Menalamba (site $\mathrm{SE}_{2}$ ), et des traces d'activités de cette espèce ont été également notées sur des tiges de bambou dans la localité de Mokaranana (site SE1). Ces deux localités sont constituées par une forêt dégradée et une forêt de savoka où se développent des bambous (Bambusa vulgaris et Cathariostachys madagascariensis). Ces types d'habitats sont appréciés par cette espèce (Wright et al. 1987, Ravaloharimanitra et al. 2011, Olson et al. 2013).

En tenant compte des résultats des inventaires dans les transects et des observations aléatoires (Prolemur simus et ses traces d'activités ainsi que les traces d'activités de Daubentonia madagascariensis), nous avons recensé dix espèces de lémuriens dans le site $\mathrm{SE}_{1}$, onze espèces dans le site $\mathrm{SE}_{2}$ et neuf espèces dans le site $\mathrm{SE}_{3}$ (Tableau 1). Concernant I'abondance, les résultats démontrent que les densités des espèces de lémuriens varient dans les trois sites d'études. Ainsi, la densité est presque nulle pour Allocebus trichotis et Cheirogaleus crossleyi ; elle varie entre zéro et deux $\mathrm{i} / \mathrm{km}^{2}$ pour Lepilemur mustelinus et Daubentonia madagascariensis ; elle varie entre zéro et trois i $/ \mathrm{km}^{2}$ pour Cheirogaleus major, Eulemur rubriventer et Propithecus diadema. Pour le cas d'Indri indri, elle varie entre un et quatre $\mathrm{i} / \mathrm{km}^{2}$ et elle est comprise entre deux et sept $\mathrm{i} / \mathrm{km}^{2}$ pour Microcebus lehilahytsara, entre cinq et seize $\mathrm{i} / \mathrm{km}^{2}$ pour Avahi laniger, entre un et vingt $\mathrm{i} / \mathrm{km}^{2}$ pour Hapalemur griseus et entre cinq et quarante-deux $\mathrm{i} / \mathrm{km}^{2}$ pour Eulemur fulvus.

NOTRE ÉTUDE DES LÉMURIENS DANS LA ZONE DE TOROTOROFOTSYPARRAPPORTAUXTRAVAUXANTÉRIEURS. En 1999, les études effectuées dans le cadre de la déclaration du marais de Torotorofotsy en "site Ramsar » ont dénombré 11 espèces de lémuriens, à savoir : Avahi laniger, Cheirogaleus major, Daubentonia madagascariensis, Eulemur fulvus, E. rubriventer, Hapalemur griseus, Indri indri, Lepilemur mustelinus, Microcebus rufus, Propithecus diadema et Varecia variegata variegata (Zimmermann et Andrianarivo 2000). En 2004, les études d'impact environnemental liées à l'implantation du projet d'exploitation de nickel et cobalt d'Ambatovy dans la zone de Torotorofotsy ont inventorié sept espèces : Avahi laniger, Cheirogaleus major, Eulemur fulvus, E. rubriventer, Hapalemur griseus, Microcebus rufus et Propithecus diadema (Ambatovy Project 2006). Outre ces études, en 2004, I'équipe de I'Association Mitsinjo a recensé pour la première fois la présence de Prolemur simus à Torotorofotsy et, en 2008, la présence des indices d'activités d'autres groupes (Dolch et al. 2004, 2008). Dans cette 
étude, 13 espèces ont été inventoriées, deux espèces nouvellement recensées, donc, en plus des onze espèces déjà connues: Cheirogaleus crossleyi et Allocebus trichotis. Dans nos inventaires, l'espèce Varecia $v$. variegata n'a été relevée dans aucun des trois sites d'observation et les microcèbes rencontrés appartiendraient à l'espèce Microcebus lehilahytsara (Roos et Kappeler 2006) mais pas à M. rufus (Mittermeier et al. 2006). Nous avons remarqué dans le site $\mathrm{SE}_{2}$, la présence de deux morphotypes de Hapalemur griseus qui vivent en sympatrie. Le pelage du premier est gris roux sur la zone frontale et sur la partie dorsale tandis que la partie ventrale est grise clair, la queue de couleur gris foncé est touffue, le museau de couleur noir est court. Le second type a une taille plus petite que le premier. II présente un dimorphisme sexuel distinctif, c'est-à-dire que le mâle a un pelage uniforme gris foncé, alors que la femelle est gris cendré et présente une face ventrale gris-blanchâtre. À noter que ces variations morphotypiques d'Hapalemur existent également dans la forêt de Maromizaha qui se trouve à $10 \mathrm{~km}$ à l'est de Torotorofotsy (Rakotosamimanana et al. 2004).

CARACTÉRISTIQUES DES LÉMURIENS DANS LE MARAIS DE TOROTOROFOTSY ET DE SES BASSINS VERSANTS PAR RAPPORT AUX AUTRES AIRES PROTÉGÉES. En comparant la richesse de Torotorofotsy en lémuriens avec d'autres aires protégées comme le parc de Zahamena qui abrite 13 espèces de lémuriens (Rakotoarison et al. 1997), Mantadia qui compte 11 espèces (Groves 2000), AnalamazaotraAndasibe qui abritent 12 espèces (Groves 2000), le Parc National d'Andringitra (Goodman et Rasolonandrasana 2001) et celui du Parc National de Ranomafana (Wright et al. 1987, Goodman et Rasolonandrasana 2001) qui en comptent chacun 12 espèces, nous constatons que Torotorofotsy présente une richesse élevée et une diversité spécifique de lémuriens analogues à celles de ces autres aires protégées qui sont localisées dans les forêts denses humides sempervirentes de moyenne altitude du versant oriental de l'île.

LES EFFETS DES ACTIVITÉS HUMAINES SUR LA FORÊT ET AUTRES RESSOURCES NATURELLES À TOROTOROFOTSY. Menalamba est le seul fokontany (quartier constitué par un ensemble de hameaux) prédominant dans le site de Torotorofotsy. Ce fokontany et ses environs comptent 1290 habitants (d'après enquête et recensement : 305 habitants dans la zone SE1, 585 habitants dans la zone SE2 et 400 habitants dans la zone SE3) (Fokontany Menalamba 2007). L'application de la méthode "MARP de type exploratoire», nous a permis de mener une enquête auprès de 82 ménages dans les trois sites (21 ménages dans le site $\mathrm{SE}_{1}, 40$ ménages dans le site $\mathrm{SE}_{2}$ et 21 ménages dans le site $\mathrm{SE} \mathrm{E}_{3}$ ). Les résultats de cette enquête nous ont permis d'apprendre que les activités quotidiennes de la population se concentrent sur I'agriculture, le bûcheronnage, l'artisanat, la pêche et l'élevage. L'étude de ces activités permet de déduire les pressions existantes sur les ressources naturelles, en particulier leurs effets sur les ressources forestières ainsi que sur la forêt en tant qu'habitat des lémuriens (Tableau 2). Dans le site $\mathrm{SE}_{1}$, la pression exercée sur le milieu forestier est inférieure à celle exercée sur les sites $\mathrm{SE}_{2}$ et $\mathrm{SE}_{3}$. De même, celle exercée sur les ressources du site $\mathrm{SE}_{3}$ est inférieure à celle du site $\mathrm{SE}_{2}$. Nous pouvons donc dire que le site $\mathrm{SE}_{2}$ est le plus vulnérable des trois sites.
RELATION ENTRE LES PRESSIONS

ANTHROPIQUES ET LES DENSITÉS DE LÉMURIENS.

Comme dans toute communauté paysanne, les activités de la vie quotidienne dépendent des ressources naturelles et de la qualité du terrain exploitable de la localité. Dans la zone concernée par notre étude, nous avons pu constater que plus le nombre d'habitants est élevé, plus la surface dégradée de I'habitat forestier s'agrandit (Tableau 3).

D'après ces résultats, nous avons pu dresser une liste des facteurs générant une perturbation de l'écosystème forestier et marécageux du site de Torotorofotsy. Les feux non contrôlés, par exemple, provoquent un dégât irréversible dans les zones forestières des trois sites; ils sont liés à la «tradition de feu » par la pratique du tavy (culture sur brûlis pour l'exploitation du riz), et s'ajoutent parfois à la pratique de l'agriculture vivrière et à la construction de campements temporaires (lasy) ou de campements permanents (potro). En plus de ces activités, il faut compter l'exploitation directe des ressources forestières et la chasse d'animaux sauvages. Les différents facteurs énumérés ci-dessus peuvent influencer les ressources naturelles et la densité des lémuriens qu'on observe dans le site en raison de la fragmentation de leurs habitats qu'ils impliquent (Andriamasimanana et al. 2001, Lehman et al. 2006).

ABONDANCE ET INDICE DE STABILITÉ DES

LÉMURIENS DANS LA ZONE DE TOROTOROFOTSY. La présence des espèces de lémuriens pendant nos observations reposait sur le hasard de l'échantillonnage et sur l'effet des facteurs écologiques que les différents types de micro-habitats de Torotorofotsy subissaient au moment de notre étude. Comme les densités relatives calculées sont des nombres fractionnés, en arrangeant par ordre prioritaire les densités relatives de chaque espèce, nous obtenons un classement de densité des lémuriens dans chaque site (Tableau 4). À partir de ce classement de densités, nous pouvons calculer la moyenne de l'indice de stabilité (M).

Le classement de ces indices définit une hiérarchisation de stabilité des espèces de la plus stable vers la moins stable dans l'ensemble de la zone. Le résultat de ce calcul de stabilité démontre que $M \in[12,10]$ pour les espèces Eulemur fulvus et Hapalemur griseus, elle sont donc considérées comme des espèces "plus stables ». Puis $M \in[9.99,8]$ pour les Avahi laniger et Microcebus lehilahytsara, ces espèces sont dites «stables ». $M \in[7.99,3]$ pour Lepilemur mustelinus, Indri indri, Propithecus diadema, Cheirogaleus crossleyi et C. major, elles sont considérées comme des espèces "peu stables ». Enfin, les espèces "moins stables" dont $\mathrm{M} \in[2.99,0]$ sont Allocebus trichotis, Daubentonia madagascariensis et Eulemur rubriventer. En terme de sensibilité par rapport à l'abondance relative de l'espèce et à la conservation des lémuriens dans la zone de Torotorofotsy et de ses bassins versants, ces constatations permettent de déduire que les deux espèces Eulemur fulvus et Hapalemur griseus sont les lémuriens les moins sensibles, que Avahi laniger et Microcebus lehilahytsara sont peu sensibles, que Cheirogaleus crossleyi, c. major, Indri indri, Lepilemur mustelinus et Propithecus diadema constituent les espèces sensibles, et que Allocebus trichotis, Daubentonia madagascariensis et Eulemur rubriventer sont les espèces les plus sensibles (Figure 2). 
TABLEAU 2. La répartition des différentes pressions recensées dans les trois sites d'étude de Torotorofotsy et ses bassins versants. Site SE 1 : Mandaniresaka

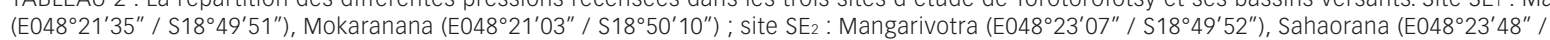

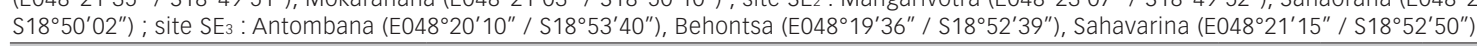

\begin{tabular}{|c|c|c|c|c|c|}
\hline $\begin{array}{l}\text { Catégorisation des activités dans les } \\
\text { zones forestières }\end{array}$ & $\begin{array}{l}\text { Types d'activités identifiés entrainant des perturbations dans les zones } \\
\text { forestières }\end{array}$ & $\mathrm{SE}_{1}$ & $\mathrm{SE}_{2}$ & $\mathrm{SE}_{3}$ & Total \\
\hline Usage de feux non contrôlés & Feux sauvages (ha) & 34 & 92 & 40 & 166 \\
\hline Usage de feux pour l'agriculture & Défrichement des zones forestières, tavy (ha) & 16 & 30 & 10 & 56 \\
\hline \multirow{2}{*}{$\begin{array}{l}\text { Usage de feux pour l'agriculture et } \\
\text { habitation près de la forêt }\end{array}$} & Accaparement de terrain agricole et campement temporaire, lasy (ha) & 2 & 4 & 2 & 8 \\
\hline & Accaparement de terrain agricole et campement définitif, potro (ha) & 4 & 8 & 6 & 18 \\
\hline \multirow{5}{*}{$\begin{array}{l}\text { Défrichement dans les zones } \\
\text { forestières }\end{array}$} & $\begin{array}{l}\text { Abattage d'arbres forestiers (diamètre hauteur de poitrine > } 12 \mathrm{~cm} \text {; } \\
\text { nombre de pieds) }\end{array}$ & 0 & 22 & 40 & 66 \\
\hline & Cheval de sciage pour bois (nombre de pieds) & 0 & 1 & 5 & 6 \\
\hline & Collecte de feuilles de Pandanus (nombre de pieds) & 15 & 3 & 1 & 19 \\
\hline & Collecte de fibres et écorces végétales (nombre de pieds) & 4 & 3 & 0 & 7 \\
\hline & Collecte de miel (nombre de pieds) & 6 & 0 & 3 & 9 \\
\hline \multirow{3}{*}{ Chasse } & Pièges pour autres animaux (piège) & 5 & 4 & 2 & 11 \\
\hline & Piège à lémuriens (piège) & 0 & 5 & 3 & 8 \\
\hline & Chasse observéé dans la forêt (nombre de groupes) & 0 & 2 & 0 & 2 \\
\hline Élevage & Pacage des bœufs (nombre de troupeaux) & 3 & 1 & 5 & 9 \\
\hline Total des activités identifiées & 13 & 9 & 12 & 11 & \\
\hline
\end{tabular}

STRATÉGIE ET PRIORISATION DES ACTIONS DE CONSERVATION DES LÉMURIENS ET DE LEURS HABITATS. Dans la zone de Torotorofotsy et de ses bassins versants, les trois sites $\mathrm{SE}_{1}, \mathrm{SE}_{2}$ et $\mathrm{SE}_{3}$ devraient être considérés pour la richesse de leur habitat forestier et des espèces qu'ils abritent dans la cadre des priorités en matière de conservation de la nature. En effet, les sites prioritaires sont déterminés en fonction des critères d'identification des zones prioritaires de conservation établis à partir de la présence d'espèces endémiques locales, spécifiques et représentatives du milieu dont la qualité de I'habitat présente un niveau de menace tolérable (TNC 2003) (Tableau 5).

Selon les caractéristiques des trois sites d'étude de Torotorofotsy, le site SE2 (Mangarivotra -Sahaorana) paraît être le site le plus favorable à la conservation des lémuriens, suivi par le site $\mathrm{SE}_{1}$ (Mandaniresaka - Mokaranana) et, enfin, par le site $\mathrm{SE}_{3}$ (Antombana - Behontsa - Sahavarina).

\section{DISCUSSION}

Plusieurs thèmes peuvent être des sujets de discussions dans cette étude, mais nous essayerons de nous cantonner à la relation entre les ressources naturelles et les facteurs anthropiques.

NOMBRE POTENTIEL D'ESPÈCES DE LÉMURIENS DANS

LA ZONE DE TOROTOROFOTSY ET SES BASSINS VERSANTS. Les différentes études effectuées à Torotorofotsy démontrent que ce site Ramsar peut contenir 14 espèces de lémuriens,

TABLEAU 3 . Variation des surfaces forestières dégradées en fonction du nombre des habitants dans les sites SE1, $\mathrm{SE}_{2}$ et $\mathrm{SE}_{3}$ (légende conforme au Tableau 2).

\begin{tabular}{lccc}
\hline $\begin{array}{l}\text { Catégorisation des activités humaines dans les } \\
\text { zones forestières }\end{array}$ & $\mathrm{SE}_{1}$ & $\mathrm{SE}_{2}$ & $\mathrm{SE}_{3}$ \\
\hline Usage de feux non contrôlés (ha) & 34 & 92 & 40 \\
\hline Usage de feux pour l'agriculture, tavy (ha) & 16 & 30 & 10 \\
\hline $\begin{array}{l}\text { Usage de feux dans l'agriculture et habitation } \\
\text { près de la forêt, acquisition de terrain (ha) }\end{array}$ & 6 & 12 & 8 \\
\hline $\begin{array}{l}\text { Totales des surfaces dégradées (ha) } \\
\text { Taux de dégradation par rapport à la surface de }\end{array}$ & $57 \%$ & $137 \%$ & $59 \%$ \\
$\begin{array}{l}\text { Torotorofotsy (\%) } \\
\text { Nombre d'habitants }\end{array}$ & & & \\
\hline
\end{tabular}

à savoir: Allocebus trichotis, Avahi laniger, Cheirogaleus crossleyi, C. major, Daubentonia madagascariensis, Eulemur fulvus, E. rubriventer, Hapalemur griseus, Indri indri, Lepilemur mustelinus, Microcebus lehilahytsara, Prolemur simus, Propithecus diadema et Varecia variegata. Mais depuis l'inventaire de 2004 (Ambatovy Project 2006) et pendant la présente étude, nous avons noté I'absence de Varecia variegata. Cette absence peut s'expliquer par une intolérance de cette espèce aux différents facteurs anthropiques entrainant la diminution de son micro-habitat car cette espèce a une préférence pour le support : arbre à haute canopée (Ratsimbazafy 2002), alors que ces arbres sont déjà exploités. II n'est pas impossible non plus que les groupes qui existaient dans la partie nord-est (site $\left.\mathrm{SE}_{2}\right)$ ont été chassés.

PRÉSENCE DE DEUX MORPHOTYPES DE HAPALEMUR GRISEUS. En raison de la potentielle richesse d'espèces de lémuriens, nous avons observé, pendant cette étude, la présence de deux morphotypes de Hapalemur griseus. Une question se pose face à cette observation : s'agit-il d'une indication que le site est une zone de croisement de répartition biogéographique ou s'agit-il d'une source d'endémisme de

TABLEAU 4 . Rang de la densité des lémuriens dans les sites $\mathrm{SE}_{1}, \mathrm{SE}_{2}$ et $\mathrm{SE} 3$ (légende conforme au Tableau 2).

\begin{tabular}{lccc}
\hline Espèces de lémuriens & $\mathrm{SE}_{1}$ & $\mathrm{SE}_{2}$ & $\mathrm{SE}_{3}$ \\
\hline Allocebus trichotis & 3 & 1 & 1 \\
\hline Daubentonia madagascariensis & 5 & 1 & 1 \\
\hline Cheirogaleus major & 6 & 1 & 1 \\
\hline Eulemur rubriventer & 1 & 6 & 1 \\
\hline Cheirogaleus crossleyi & 1 & 3 & 5 \\
\hline Lepilemur mustelinus & 4 & 4 & 4 \\
\hline Propithecus diadema & 1 & 7 & 5 \\
\hline Indri indri & 7 & 4 & 7 \\
\hline Microcebus lehilahytsara & 8 & 8 & 9 \\
\hline Avahi laniger & 9 & 9 & 11 \\
\hline Hapalemur griseus & 10 & 11 & 8 \\
\hline Eulemur fulvus & 11 & 10 & 10 \\
\hline
\end{tabular}




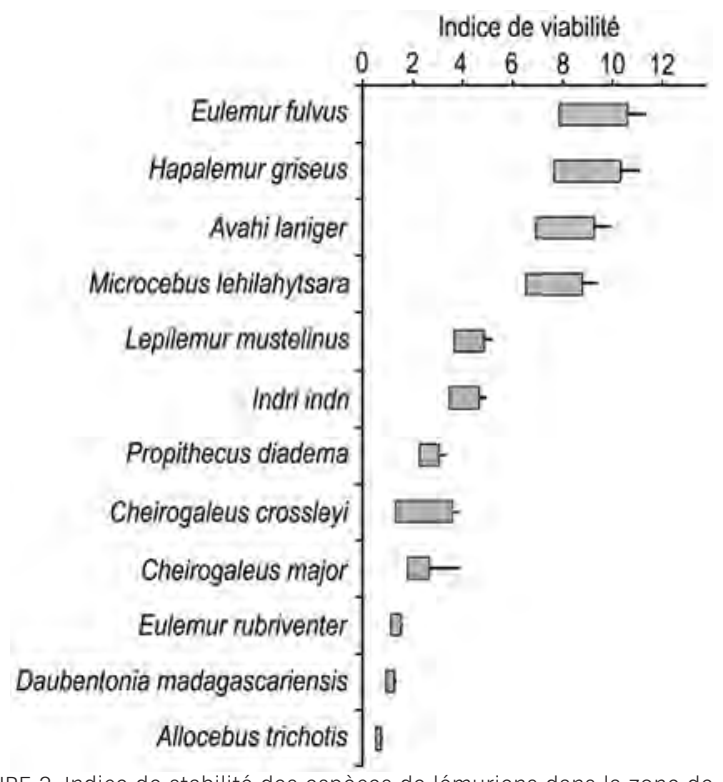

FIGURE 2. Indice de stabilité des espèces de lémuriens dans la zone de Torotorofotsy et ses bassins versants en fonction des facteurs écologiques existants.

cette espèce ? Hapalemur griseus est une espèce territoriale et le groupe a un système d'accouplement monogame ou polygyne ; ces comportements sociaux reproductifs sont probablement dus à la défense des ressources et à la défense des femelles (Tan 1999, Mutschler et al. 2000). Par conséquent, l'échange de génotypes entre les deux sous-espèces est limité par ces caractères comportementaux et la naissance d'une population hybride est improbable. L'analyse génétique de ces deux morphotypes de Hapalemur griseus pourrait déterminer s'il existe une variation spécifique ou non. II semble que Hapalemur griseus, qui est une espèce peu sensible dans cette zone, s'adapte bien aux facteurs anthropiques dans le milieu où il existe actuellement (Andriamasimanana et al. 2001, Irwin et al. 2005, Lehman et al. 2006).
MODE DE GESTION DES RESSOURCES NATURELLES DANS LA ZONE DE TOROTOROFOTSY. Cette étude démontre que I'Homme dépend de son environnement auquel il a attaché ses valeurs culturelles. Dans le site de Torotorofotsy, I'utilisation des feux et d'autres moyens similaires semble être le principal facteur de pression faisant régresser la superficie des zones forestières existantes et les fragmente. Les autres moyens d'exploitations des ressources peuvent être maîtrisés par des engagements entre les communautés ou bien par l'application des lois imposées par les autorités compétentes. Le présent cas étudié dévoile l'atténuation de la conservation des ressources naturelles dans cette zone. Trois types de gestions de ressources naturelles sont respectés et suivis par les habitants de Torotorofotsy. Deux de ces types de gestions sont axés sur des ressources aquatiques: les poissons et les cypéracées. Ainsi, la pêche et la collecte de ces plantes aquatiques pour la confection de natte est censée s'arrêter pendant la saison des pluies (fermeture de collecte des cypéracées ou mihidy rambo en décembre et ouverture de la pêche ou vaky sihitry en mai). Le troisième type de gestion consiste en l'interdiction de la chasse des lémuriens, définis comme fady, c'est-à-dire tabou, pour tous les habitants qui résident dans le terroir de Torotorofotsy. Ces interdictions sont dictées dans des lois conventionnelles (Dinam-pokonolona) établies entre les fokonolona (tous les habitants du fokontany) depuis I'année 2005 (année de déclaration de Torotorofotsy en site Ramsar). Ces interdictions sont généralement respectées, mais les délinquants existent toujours dans le fokontany même ou bien venant d'autres fokontany périphériques. Pendant les inventaires, nous avons rencontré des chasseurs dans le site SE2 et nous avons vu des pièges dans les sites $\mathrm{SE}_{2}$ et $\mathrm{SE}_{3}$. Force est de constater que la chasse n'est pas totalement éradiquée dans la zone et les résultats des enquêtes nous révèlent que de nombreuses familles (environ $70 \%$ des ménages interrogés) apprécient et mangent encore les animaux sauvages, appelés haza ou

\begin{tabular}{|c|c|c|c|}
\hline Sites d'étude & Critères de I'UICN & $\begin{array}{l}\text { Activités anthropiques / Pression } \\
\text { existante }\end{array}$ & $\begin{array}{l}\text { Diversité spéci- } \\
\text { fique en lémuriens }\end{array}$ \\
\hline \multirow[t]{5}{*}{$\mathrm{SE}_{2}$} & $\begin{array}{l}\text { Présence d'espèce menacée : En danger critique (CR): Prolemur simus; En } \\
\text { danger (EN) : Daubentonia madagascariensis, Indri indri, Propithecus } \\
\text { diadema }\end{array}$ & Défrichement et tavy & 11 \\
\hline & $\begin{array}{l}\text { Présence d'espèces Données insuffisantes (DD) : Cheirogaleus crossleyi, } \\
\text { Lepilemur mustelinus, Microcebus lehilahytsara }\end{array}$ & Défrichement et campement & \\
\hline & & $\begin{array}{l}\text { Défrichement et exploitations } \\
\text { forestières }\end{array}$ & \\
\hline & & Autres pièges - pièges à lémurien & \\
\hline & & Chasse & \\
\hline \multirow[t]{4}{*}{$\mathrm{SE}_{1}$} & Présence d'espèce En danger critique (CR) : Prolemur simus & Défrichement tavy & 10 \\
\hline & en danger (EN) : Daubentonia madagascariensis, Indri indri & Défrichement et campement & \\
\hline & $\begin{array}{l}\text { Présence des espèces Données insuffisantes (DD) : Allocebus trichotis, } \\
\text { Lepilemur mustelinus, Microcebus lehilahytsar }\end{array}$ & $\begin{array}{l}\text { Défrichement / exploitations } \\
\text { forestières }\end{array}$ & \\
\hline & & Autres pièges & \\
\hline \multirow[t]{4}{*}{$\mathrm{SE}_{3}$} & $\begin{array}{l}\text { Présence d'espèce En danger (EN) : Daubentonia madagascariensis, } \\
\text { Indri indri, Propithecus diadema }\end{array}$ & Défrichement tavy & 9 \\
\hline & $\begin{array}{l}\text { Présence des espèces Données insuffisanets (DD) : Cheirogaleus crossleyi, } \\
\text { Lepilemur mustelinus, Microcebus lehilahytsara }\end{array}$ & Défrichement et campement & \\
\hline & & $\begin{array}{l}\text { Défrichement / exploitations } \\
\text { forestières }\end{array}$ & \\
\hline & & Autres pièges - Pièges à lémurien & \\
\hline
\end{tabular}


« viande de brousse » (oiseaux, petits mammifères, mammifères carnivores, lémuriens, potamochères, etc.). À Torotorofotsy, la chasse du gibier est destinée à la consommation familiale et sert à satisfaire les apports en protéine des habitants (Jenkins et al. 2011) et les chasseurs ne sélectionnent pas les animaux à chasser, comme dans la zone de Makira, où les chasseurs ont des préférences pour les lémuriens (Rasolofoson 2007b, Golden 2009). Néanmoins, à cause du fady, les Betsimisaraka ne chassent jamais des babakoto (Indri indri).

Ainsi, la sensibilisation doit être axée sur la maîtrise du feu, qui est prisé pour transformer une zone forestière en terrain agricole, car ce facteur est le premier déstabilisateur de l'écosystème forestier (Kiener 1963, Rasoavarimanana 1997, Aubert et al. 2003). Quant au tavy, la gestion de la fertilité des terres entraîne écologiquement un dynamisme des formations végétales secondaires qui évoluent vers une formation climacique appelée savoka et qui sera à nouveau exploitable après cinq à dix ans de jachère (Kiener 1963, Aubert et al. 2003, Styger et al. 2007). Ce dynamisme de formation végétale entraine un dynamisme d'éparpillement des hameaux en fonction des terrains fertiles.

L'exploitation de la mine à Ambatovy et la construction du pipeline le long du marais entrainent également, sur le plan écologique, une fragmentation de différents habitats forestiers et marécageux. Les fragmentations des habitats forestiers entrainent parfois l'isolement de groupes de lémuriens et affaiblissent leurs chances de survie (Lehman et al. 2006). Plusieurs groupes d'animaux vont subir les conséquences de ce changement de territoire et pourront, ou non, tolérer et s'adapter à la modification de leur domaine vital (Andriamasimanana et al. 2001, Irwin et al. 2005). Les travaux de la mise en place du pipeline et des infrastructures du projet Ambatovy ont commencé en décembre 2008, pendant cette étude. En 2013, les infrastructures sont mises en place; le pipeline facilite l'accès au site de Torotorofotsy en permettant une voie de communication pour tout public. Sur le plan social, I'implantation du projet Ambatovy a accaparé une partie des terrains agricoles. Pour les gens de la région, ce concept reflète la mise en place de nouvelles règles de gestion de l'espace et des ressources naturelles impliquées par la création de ce pôle d'attraction économique (Phillips 2002, UICN 2003). La perception des populations de Torotorofotsy est généralement dominée par une image d'invasion de leur territoire par des personnes venant de l'extérieur qui leur imposent des règles et des interdits.

\section{CONCLUSION}

La mise en œuvre du plan de gestion pour la conservation de la forêt des bassins versants de Torotorofotsy appelle à la sauvegarde des habitats naturels des lémuriens existants dans ce site Ramsar. Cette zone abrite actuellement 13 espèces de lémuriens pour lesquelles elle représente une niche écologique importante car ses blocs forestiers sont connectés avec le corridor forestier Ankehiheny-Zahamena (CAZ). Cette connexion forestière offre un écosystème potentiel pour ces espèces et est favorable aux échanges de flux génétiques nécessaires à leur pérennité. Concernant la conservation des lémuriens dans cette zone, leur rang de viabilité montre que les efforts doivent examiner en priorité les espèces menacées de faible densité aux statuts de En danger critique d'extinction, En danger ou Vulnérable; selon les statuts de menace déterminés par
I'UICN et selon les degrés de sensibilité, la conservation des six espèces de lémuriens suivantes sont prioritaires dans cette zone : Allocebus trichotis, Daubentonia madagascariensis, Eulemur rubriventer, Indri indri, Prolemur simus, Propithecus diadema ; puis les espèces qui ont une densité faible: Cheirogaleus major, C. crossleyi et Lepilemur mustelinus; enfin les espèces moins menacées comme Microcebus lehilahytsara, Avahi laniger, Hapalemur griseus et Eulemur fulvus.

Les gestionnaires et les différents acteurs du développement dans la zone de Torotorofotsy devront mettre en œuvre une gestion du terroir des bassins versants (ANGAP 2003) pour protéger la biodiversité exceptionnelle. Il est également nécessaire de sensibiliser la population locale et riveraine par l'application effective d'une éducation environnementale, la formation à une meilleure maîtrise du feu, principal facteur de pression sur la biodiversité du site, et à la gestion durable des ressources naturelles. Ces actions sont compatibles avec la politique environnementale que Madagascar a adoptée pour la valorisation de sa biodiversité. L'adoption de cette politique mènerait au développement durable de la zone et de la région tout en permettant la conservation des écosystèmes et le développement économique et socio-culturel.

\section{REMERCIEMENTS}

Nous tenons à remercier ici le Projet Ambatovy pour les appuis matériels et financiers qu'il a apporté pour concrétiser le présent projet, nous adressons nos remerciements à Monsieur le Directeur du Département Environnement du Projet Ambatovy et à toutes les équipes techniques ainsi que les personnels de Projet Ambatovy volet Biodiversité et Gestion Forestière pour l'aide qu'ils ont apportée à la réalisation de la mission. Nous adressons également nos remerciements aux partenaires administratifs Direction Générale des Eaux et Forêts (DGEEF), à la Direction de Préservation de la Biodiversité du Ministère de I'Environnement et des Eaux et Forêts et du Tourisme (MINENVEFT) ; à la Circonscription des Eaux et Forêts de Moramanga (CIREFT), au District de Moramanga, aux Maires, Chef de fokontany, Tangalamena, à toute l'équipe de l'Association Mitsinjo, en particulier à Jeannot et aux partenaires sur terrain (VOI), ainsi qu'aux guides et aux fokonolona pour nous avoir facilité l'accès sur le terrain et nous avoir aidé dans la réalisation du présent projet. Nous adressons nos remerciements aux rapporteurs et aux correcteurs qui nous ont permis d'améliorer considérablement cette contribution.

\section{RÉFÉRENCES}

Ambatovy Project. 2006. Environmental Assessment Ambatovy Project. Volume J: Biological appendices. The Ambatovy Project, Antananarivo. Available at <http://www.sherritt.com/doc08/files/ Ambatovy_EIA/Documents/main\%20menu\%20ENGLISH.pdf>

Andriamasimanana, R. H., Rabenandrasana, M. N., Raminoarisoa, V., The Seing, S., Virginie, M. C., Ratelolahy, F. J. et Rakotonirainy, E. O. 2001 Effets de la fragmentation de la forêt humide sur les populations d'oiseaux et des lémuriens dans le corridor Mantadia-Zahamena. Lemur News 6: 18-22.

ANGAP. 2003. Plan de gestion de Réseau National des Aires Protégées de Madagascar. ANGAP et Ministère de I'Environnement des Eaux et Forêts. $112 \mathrm{pp}$.

Aubert, S., Razafiarison, S. et Bertrand A. (eds.). 2003. Déforestation et Système Agraire à Madagascar : Les Dynamiques de Tavy sur la Côte Orientale. CIRAD, CITE, Fofifa, Madagascar. 
Dolch, R., Fiely, J. L., Ndriamiary, J. N., Rafalimandimby, J.; Randriamampionona, R., Engberg, S. E. \& Louis Jr., E. E. 2008. Confirmation of the greater bamboo lemur, Prolemur simus, north of the Torotorofotsy wetlands, eastern Madagascar. Lemur News 13: 14-17.

Dolch, R., Hilgartner, R. D., Ndriamiary, J. N. \& Randriamahazo, H. 2004. “The grandmother of all bamboo lemurs": evidence for the occurrence of Hapalemur simus in fragmented rainforest surrounding the Torotorofotsy marshes, central eastern Madagascar. Lemur News 9: 24-26.

Fokontany Menalamba, 2007. Recensement administratif de la population. Registre de recensement de fokontany Menalamba Commune Rurale Andasibe.

Ganzhorn, J. U. 1988. Food partitioning among Malagasy primates. Oecologia 75, 3: 436-450. (doi:10.1007/BF00376949)

Ganzhorn, J. U., 1994. Inventaire biologique dans la forêt de Zombitse. Les Lémuriens. In Goodman, S.M., Langrand, O. (eds), pp 70-72. Recherches pour le Développement, Série Sciences Biologiques, Numéro Spécial, CIDST, Antananarivo.

Ganzhorn, J. U., Rakotosamimanana, B., Hannah, L., et al. 1997. Priorities for biodiversity conservation in Madagascar. Primate Report, Goettingen $48,1$.

Golden, C. D. 2009. Bushmeat hunting and use in the Makira Forest, northeastern Madagascar: a conservation and livelihoods issue. Oryx 43, 3: 386-392. (doi:10.1017/S0030605309000131)

Goodman, S. M. \& Rasolonandrasana, B. P. N. 2001. Elevation zonation of birds, insectivores, rodents and primates on the slopes of the Andringitra Massif, Madagascar. Journal of Natural Histstory 35, 2: 285-305. (doi:10.1080/00222930150215387)

Green, G. M. \& Sussman, R. W. 1990. Deforestation history of the eastern rain forests of Madagascar from satellite images. Science 248 : 212-215. (doi:10.1126/science.248.4952.212)

Groves, C. P. 2000. The genus Cheirogaleus: unrecognized diversity in dwarf lemurs. International Journal of Primatology 21, 6: 943-962. (doi:10.1023/A:100555901263)

Gueye, B. \& Schoonmaker Freudenberger, K. 1991. Introduction à la Méthode Accélérée de Recherche Participative (MARP) : quelques notes pour appuyer une formation pratique. International Institute for Environment and Development. Sustainable Agriculture Program, 70 pp. Available at <http://pubs.iied.org/pdfs/6022IIED.pdf>

Irwin, M. T., Johnson, S. E. \& Wright, P. C. 2005. The state of lemur conservation in south-eastern Madagascar: Population and habitat assessments for diurnal and cathemeral lemurs using surveys, satellite imagery and GIS. Oryx 39, 2: 204-218. (doi:10.1017/S0030605305000451)

Jenkins, R. K. B., Keane, A., Rakotoarivelo, A. R., et al. 2011. Analysis of patterns of bushmeat consumption reveals extensive exploitation of protected species in eastern Madagascar. PLOS ONE 6, 12 : e27570. (doi:10.1371/journal.pone.0027570)

Kiener, A., 1963. Le "Tavy" à Madagascar ses différentes formes et dénomination. Bilan du tavy et problèmes humains. Moyens de lutte. Bois et Forêts des Tropiques 90: 9-16.

Lehman, S. M, Rajaonson, A. \& Day S. 2006. Edge effects and their influence on lemur density and distribution in southeast Madagascar. American Journal of Physical Anthropology 129, 2: 232-241. (doi:10.1002/ ajpa.20241)

Martin, P. R. \& Bateson P. P. G. 1993. Measuring Behaviour: An Introductory Guide. Cambridge University Press, Cambridge.

Ministère de l'Environnement et des Forêts / Circonscription Régionale de I'environnement et des forêts - Moramanga, Association Mitsinjo, Communauté de base Taratra et Projet Ambatovy 2009. Mise à jour du plan d'aménagement du site de Torotorofotsy. Plan d'Aménagement de Gestion Social de Torotorofotsy, 98 pp.

Mittermeier, R. A., Konstant, W. R., Hawkins, F., et al. 2006. Lemurs of Madagascar, Conservation International Tropical Field Guide Series, Washington DC.

Mittermeier, R. A., Louis Jr., E. E., Matthews, R., et al. 2010. Lemurs of Madagascar. Conservation International, Washington DC.

Mutschler, T., Nievergelt, C. M., and Feistner, A. T. C. 2000. Social organization of the Alaotra gentle lemur (Hapalemur griseus alaotrensis). American Journal of Primatology 50, 1: 9-24. (doi:10.1002/(SICI)10982345(200001)50:1<9::AID-AJP2>3.0.CO;2-2)
Olson, E. R., Marsh, R. A., Bovard, B. N., Randrianarimanana, H. L. L., Ravaloharimanitra, M., Ratsimbazafy, J. H. \& King, T. 2013. Habitat preferences of the Critically Endangered Greater bamboo lemur (Prolemur simus) and densities of one of its primary food sources, Madagascar giant bamboo (Cathariostachys madagascariensis), in sites with different degrees of anthropogenic and natural disturbance. International Journal of Primatology 34, 3: 486-499. (doi:10.1007/s10764-013-9674-z)

Peirera, M. E., Kaufman, R., Kappeler, P. M., \& Overdorff, D. J. 1990. Female dominance does not characterize all of the Lemuridae. Folia Primatologica 55, 2: 96-103. (doi:10.1159/000156505)

Phillips, A., 2002. Management guidelines for IUCN Category V Protected Areas Landscapes/Seascapes. Best Practice Protected Area Guidelines Series $n^{\circ}$ 9. IUCN Gland, Switzerland, and Cambridge, UK.

Pollock, J. 1979. Spatial distribution and ranging behaviour in lemurs. In: The Study of Prosimian Behaviour. G. A. Doyle \& R. D. Martin (eds.), pp 359-409. Academic Press, NY.

Powzyk, J. \& Thalmann, U. 2003. Indri (Indri indri). In: The Natural History of Madagascar. S. M. Goodman \& J. P. Benstead (eds.), pp 1342-1345. The University of Chicago Press, Chicago.

Rakotoarison, N., Zimmermann, H. \& Zimmermann, E. 1997. First discovery of the hairy-eared dwarf lemur (Allocebus trichotis) in a higland rain forest of eastern Madagascar. Folia Primatologica 68, 2: 86-94. (doi:10.1159/000157235)

Rakotosamimanana, B., Raharizelina, R. R., Ralisoamalala, R. C., et al. 2004. Comment et pourquoi les lémuriens diurnes disparaissent peu à peu dans les forêts d'Ambato et de Maromizaha (région de Moramanga) Madagascar? Lemur News 9: 19-24.

Ramsar. 1999. Convention on Wetlands. Evaluation des risques : Cadre d'évaluation des risques pour les zones humides. Résolution VII. 10. "L'homme et les zones humides: un lien vital". $7^{\mathrm{e}}$ Session de la Conférence des Parties contractantes à la Convention sur les zones humides, San José, Costa Rica. $11 \mathrm{pp}$.

Rasoavarimanana, M. A. 1997. Le marais d'altitude de Torotorofotsy et son environnement économique. Agriculture et Développement 14 : 3-10.

Rasolofoson, D., Rakotondratsimba, G., Rakotonirainy, O., Rasolofoharivelo, T., Rakotozafy, L., Ratsimbazafy, J., Ratelolahy, F., Andriamaholy, V. et Sarovy, A. 2007a. Le bloc forestier de Makira charnière des lémuriens. Lemur News 12: 49-53.

Rasolofoson, D., Rakotondratsimba, G., Rakotonirainy, O., Rakotozafy, L. M. A., Ratsimbazafy, J. H., Rabetafika, L. et Randrianarison, R. M. 2007b. Influences des pressions anthropiques sur les lémuriens d'Anantaka, dans la partie est du plateau de Makira, Maroantsetra, Madagascar. Madagascar Conservation \& Development 2, 1: 21-27.

Ratsimbazafy, J. 2002. Response of black-and-white ruffed lemurs (Varecia variegata variegata) to disturbance in Manombo forest Madagascar. Ph.D. thesis. State University of New York at Stony Brook.

Ravaloharimanitra, M., Ratolojanahary, T., Rafalimandimby, J., et al. 2011. Gathering local knowledge in Madagascar results in a major increase in the known range and number of sites for critically endangered greater bamboo lemurs (Prolemur simus). International Journal of Primatology 32, 3: 776-792. (doi:10.1007/s10764-011-9500-4)

Roos, C. \& Kappeler, P. M. 2006. Distribution and conservation status of two newly described cheirogaleid species, Mirza zaza and Microcebus lehilahytsara. Primate Conservation 21: 51-53. (doi:10.1896/08986207.21.1.51)

Schmid, J., 2000. Conservation planning in the Mantadia-Zahamena corridor, Madagascar. Rapid Assesment Program (RAP). Bonner Zoologische Monographien 46: 285-296.

Sterling, E. J. 2003. Daubentonia madagascariensis, aye-aye. In: The Natural History of Madagascar. S. M. Goodman \& J. P. Benstead (eds.), pp 1348-1351. The University of Chicago Press, Chicago.

Styger, E., Rakotondramasy, H. M., Pfeffer, M. J., Fernandes, E. C. M. \& Bates, D. M. 2007. Influence of slash-and-burn farming practices on fallow succession and land degradation in the rainforest region of Madagascar. Agriculture, Ecosystems \& Environment 119, 3-4: 257-269. (doi:10.1016/j.agee.2006.07.012)

Tan, C. L. 1999. Group composition, home range size, and diet of three sympatric bamboo lemur species (genus Hapalemur) in Ranomafana National Park, Madagascar. International Journal of Primatology 20, 4: 547-566. (doi:10.1023/A:1020390723639) 
TNC. 2003. The Five-S Framework for Site Conservation. The Nature Conservancy. Available at <http://www.ecology.ethz.ch/education/ Conservation_stuff/TNC_2003_5s_framework.pdf>.

UICN. 2003. Catégories et critères de I'UICN pour la Liste Rouge. Version 3.1. In: Commission de la sauvegarde des espèces, UICN - Union Mondiale pour la Nature (ed.), pp 1-23. IUCN, Gland, Switzerland, and Cambridge, UK.

Wright, P. C., Daniels, P. S., Meyers, D. M., Overdoff, D. J. \& Rabesoa, J. 1987 A census and study of Hapalemur and Propithecus in south-eastern Madagascar. Primate Conservation 8: 84-87.

Zimmermann, H. et Andrianarivo, C. 2000. La protection de la biodiversité des forêts et marais de Torotorofotsy à Andasibe - Madagascar. In : Biodiversité et Endémisme à Madagascar. W. R. Lourenco et S. M. Goodman (eds.), pp 261-272. Société de Biogéographie, Paris.
MATÉRIEL SUPPLÉMENTAIRE.

DISPONIBLE EN LIGNE UNIQUEMENT.

ANNEXE 1. Méthodes Accélérées de Recherche Participative (MARP) appliquées à l'étude de la conservation des lémuriens du site Ramsar de Torotorofotsy. 